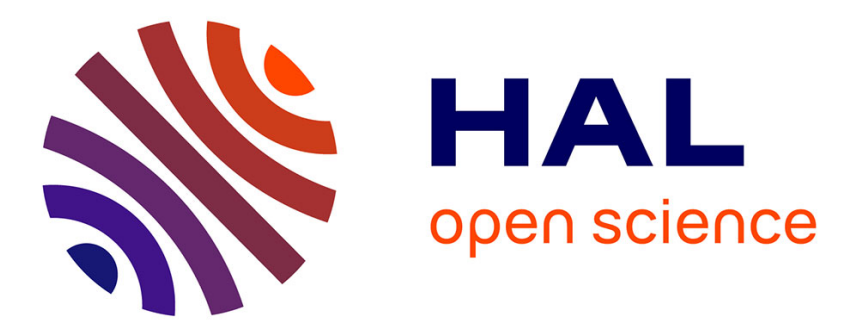

\title{
The impact of environmental changes upon the microrheological response of adherent cells
}

\author{
Cyril Picard, Athene Donald
}

\section{To cite this version:}

Cyril Picard, Athene Donald. The impact of environmental changes upon the microrheological response of adherent cells. European Physical Journal E: Soft matter and biological physics, 2009, 30, pp.127-134. 10.1140/epje/i2009-10473-y . hal-01267689

\section{HAL Id: hal-01267689 \\ https://hal.science/hal-01267689}

Submitted on 4 Feb 2016

HAL is a multi-disciplinary open access archive for the deposit and dissemination of scientific research documents, whether they are published or not. The documents may come from teaching and research institutions in France or abroad, or from public or private research centers.
L'archive ouverte pluridisciplinaire HAL, est destinée au dépôt et à la diffusion de documents scientifiques de niveau recherche, publiés ou non, émanant des établissements d'enseignement et de recherche français ou étrangers, des laboratoires publics ou privés. 


\title{
The impact of environmental changes upon the microrheological response of adherent cells
}

\author{
Cyril Picard ${ }^{a}$ and Athene Donald \\ Biological and Soft Systems, Cavendish Laboratory, University of Cambridge, Cambridge CB3 OHE, UK
}

\begin{abstract}
The mechanical behaviour of adherent cells cultured in vitro is known to be dependent on the mechanical properties of the substrate. We show that this mechanical behaviour is also strongly affected by the cells' environment. We focus here on the impact of temperature and $\mathrm{pH}$. Experiments carried out on individual cells in a tuneable environment reveal that the intra-cellular mechanical behaviour exhibits large and fast changes when the external cell environment is changed. Fast passive microrheometry measurements allow for the precise characterisation of the transient regime observed during a temperature drop. When maintained at a non physiological temperature, the cells reach a stabilised state distinct from the state observed in physiological conditions. The perturbation can be reversed but exhibits hysteretic behaviour when physiological conditions are restored. The transient regime observed during the recovery process is found to be different from the transient regime observed when leaving physiological conditions. A modified generalized Stokes-Einstein equation taking into account the cell activity through an effective temperature is proposed here to fit the experimental results. Excellent agreement between the model and the measurements is obtained for time lags from $10^{-3}$ to $1 \mathrm{~s}$ considered in this study.
\end{abstract}

PACS. 87.17.Rt Cell adhesion and cell mechanics - 87.16.dm Mechanical properties and rheology 87.16.Ln Cytoskeleton

\section{Introduction}

Living cells are known to behave as highly dynamical materials $(1 ; 2)$. Adapting their shape and mechanical properties according to their environment cells exhibit a subtle combination of active and passive behaviours (3). Through efficient mechanotransduction, cells can sense the mechanical properties of their environment and reorganize the structure and the mechanical properties of their cytoskeleton on a broad range of length scales and time scales (4). These time scales range from a few tens of milliseconds for the quick polymerisation of actin filaments over typical lengths of a couple of microns, to a few hours for cell division corresponding to reorganisation at the cell scale (5). Complex transient regimes of reorganisation of the cytoskeleton can thus be observed as a result of a sudden dynamical perturbation of the environment. Aging of the cytoskeleton under large strain $\lambda_{\lambda}$ and rejuvenation when the stress is relaxed constitute typical examples of the nonlinear dynamical responses that can be expected from a cell $(6 ; 7)$.

In its natural environment, an adherent cell is generally surrounded by complex 3D biological tissue. Chemical, as well as mechanical interactions at the interface between this tissue and the cell, drive its growth, its structure and

\footnotetext{
a Present address: Centre for Mathematical Sciences, Cam-
} bridge CB3 0WA, UK more generally its behaviour. To mimic this complex system cells are commonly grown on a $2 \mathrm{D}$ culture substrate. Although simple this in vitro reconstitution allows one to draw important conclusions concerning the cell mechanics: unlike a cell in suspension, the interactions at the interface between the cell membrane and the substrate are indeed preserved. For instance it is now accepted that the rigidity of adherent cells correlates with the rigidity of the substrate (8).

Numerous techniques have been developed to characterise the cell mechanical properties $(9 ; 10)$. Few of them, however, are suitable for a local and fast characterisation of the intracellular mechanical properties of the cell, which is known to present local and fast-changing differences (11).

Passive intracellular microrheology, based on the quantification of the random motion of intracellular tracers, has been used for more than one decade to probe the local cell properties (12). The lack of knowledge on the forces resulting from the cell activity which may perturb the thermal motion of the tracers of interest, has progressively been considered as a drawback of this technique to which techniques based on external controlled active forces are often now preferred. However passive microrheology remains one of the less invasive method to probe the cell, due to the small size of the particles that can be used and the absence of any magnetic field or laser beam focused on the cell to manipulate the particles in an active man- 
ner. Even if magnetic or optical tweezers are non-intrusive from a mechanical point of you they may perturb the cell behaviour (13). Because passive microrheology is known to be an out-of-equilibrium methodit gives insight on both the mechanical properties of the cell in the linear regime and its activity (14).

Several studies based on averages on different groups of cells have already demonstrated the importance of the stiffness and the functionalisation of the cell substrate with or without the injection of specific drugs to control the cell constituents $(15 ; 4)$. We will focus here on the local behaviour of individual adherent cells when varying fundamental parameters such as $\mathrm{pH}$ and temperature whose impact on the cell mechanics has been almost never studied to our knowledge $(16 ; 17)$. In order to control precisely these parameters, a home-made environmental chamber has been designed to be able to culture living cells for long time experiments on a microscope stage. While keeping cells in a sterile environment, this microincubator is also particularly useful for controlling and modifying the environment in which the cells sit. With the use of this chamber we can examine the potential impact on the cell of a sudden perturbation of temperature or $\mathrm{pH}$. Passive microrheological measurements based on the random motion of small beads taken up into the cells are carried out in real time to probe the cell behaviour while the environment is changing. We thus access a transient regime, during which the cell contents exhibits changes in response to the environmental perturbation. These measurements are analysed in the light of a modified generalised StokesEinstein relation, using an effective temperature to consider the dependence of the beads' motion on the cell activity, whatever its nature: traffic of molecular motors or filaments poly-depolymerisation (14).

\section{Material and method}

\subsection{Micro-incubator}

A modular micro-incubator has been developed to be able to culture cells for long time experiments under the microscope. This chamber is based on a silicon cone (flexiPERM ConA, Greiner bio one), mounted inside a stainless steel cylindrical holder. A coverslip, used as the cell substrate is inserted between the cone and the metallic holder. On the opposite side of the cone a permeable membrane (biofolie Greiner bio one) is clamped between two conical rings pressed against the cone wall. Cells are introduced via small capillaries in the sterile limited volume between the coverslip, the cone and the permeable membrane. On the other side of the permeable membrane there is a (notnecessarily sterile) atmosphere, whose gas composition is well defined and which is constantly circulated above the membrane in order to control the medium $\mathrm{pH}$. In order to control precisely the system temperature a Pt1000 sensor has been added as an extension of the silicon cone which is pressed against the substrate when the microincubator is closed. The measured temperature is then the same as the temperature sensed by the cell growing onto the substrate. On the opposite side of the microincubator a central hole Peltier (RH1.4-14-06L Melcor) placed on the stainless steel holder- is powered through a PID (proportional integral derivative) controller (MTTC-1410 Melcor) with a feedback based on the signal given by the temperature sensor. Because of the stainless steel holder the heat injected or absorbed by the Peltier element is driven all around the microincubator. In the stabilisedstate regime, the temperature can be adjusted with fluctuations smaller than $0.05^{\circ} \mathrm{C}$. The sterile liquid volume being sealed with the permeable membrane, the system can be used on an inverted microscope as well as on a-upright microscope once the cells have adhered to the substrate. Further details of this novel design will be described in a further publication.

To avoid any perturbations due to a transfer from the cell culture laboratory to the microscope room, the cells are grown inside the micro-incubator in the microscope room for 12 hours before the beginning of any microrheological measurements.

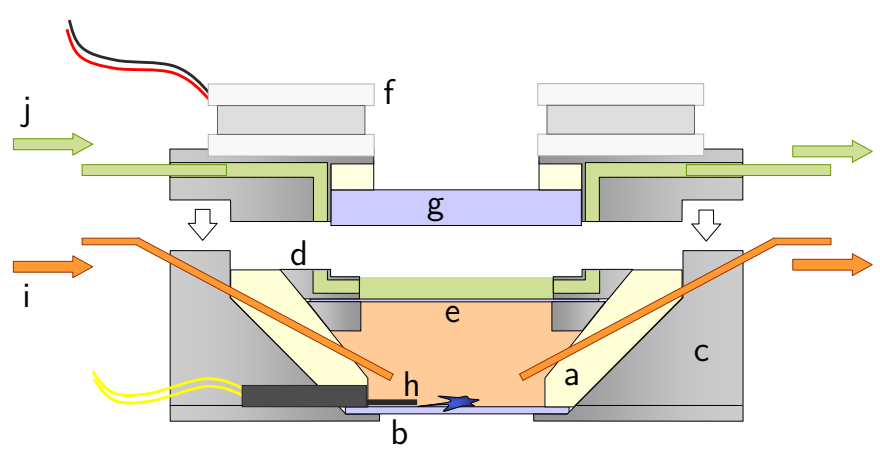

Fig. 1. Schematic of the modular microincubator. The top part has been separated from the bottom in this drawing to make it easier to understand its working: a) silicon cone, b) cover slip, c) stainless steel holder, d) clamping stainless steel rings, e) permeable membrane, f) Peltier heater, g) round glass to close the gas volume, h) temperature sensor, i) medium inlet, j) gas inlet.

\subsection{Passive intracellular microrheometry}

The cell considered in this study are standard 3T3 fibroblasts cultured in Dulbecco's Modification of Eagle's medium (DMEM) with 10\% foetal bovine serum with $1 \%$ glutamine and $1 \%$ penicillin-streptomycin in a laboratory incubator under standard cell culture conditions.

\subsubsection{Bead incorporation}

Sulphate modified polystyrene beads ( $1 \mu \mathrm{m}, 72938$ Fluka) used as tracer particles for the microrheometry are added at a volumie concentration of $510^{-6}$ in the culture medium covering the cells two days before the beginning of the 
measurements. At this stage the cells are grown in a standard T75 flask. Thanks to their negatively charged surface, the beads are easily taken up by the cells through endocytosis. This engulfment process (compared to microinjection) is part of the natural cell activity, and as such does not perturbs the cell metabolism as long as the amount of particles taken up is limited. Even if the beads were to be enclosed in endosomes, their motion depends strongly on the cytoskeleton properties (16). For such comparatively large beads, one can assume that the impact of the endosome enclosure is limited to a slight increase in the bead's diameter. Because of the relatively large size, the probed material can be treated as a continuum, which is usually one of the assumptions of one point microrheometry. Moreover beads can be observed with a moderate intensity of white light, which is not likely to perturb the cell behaviour.

One day after the beads' introduction into the T75 flask, the cells are passaged and deposited on the substrate of interest. In doing so, we remove the beads not attached to the cells and, more importantly, one observes a better spreading of the beads within each cell after this treatment: before the passage beads are mainly clustered around the nucleus whereas after the passage beads are more randomly spread within the cytosol.

\subsubsection{Bead tracking}

The beads' displacements are observed with a Zeiss LSM 510 microscope in bright field mode with a long distance $50 \mathrm{x}$ objective. The position of the beads are recorded with a video camera (Pike AVT) using the Matlab acquisition toolbox. The frame rate can reached $10^{3}$ frames per second tuning the frame size, so as to record only the region comprising the beads of interest. This simple measurement technique is one of the few allowing the simultaneous tracking of several beads, while keeping the information on the displacement of each individual bead.

A Matlab programme has been written in-house to track extremely precisely the bead's displacement. To do so, the programme determines an appropriate individual filter based on the intensity profile of each of the beads. The filtered image of each bead, appears as a white spot with a maximum intensity at the centre of the bead (intensity level 1) surrounded by a black background (intensity level 0 ). We found that the logarithm of the filtered image of each bead can be well fitted by an axisymmetric quadratic profile centred on the centre of the bead (the logarithm of the image intensity varies indeed from a maximum value at the centre of the bead to minus infinity when reaching the black background). Thanks to this fitting process based on a large number of pixels around the bead centre it is possible to minimize the sensitivity to static error due to light fluctuations (18). To avoid the problem of pixel locking, the fit is carried out on a large number of pixels around the approximate bead centre using weighting factors to decrease progressively the impact of the pixels situated further away from the bead centre.
As a result of this filtering-fitting process the bead centre can be precisely identified with a resolution better than $2 \mathrm{~nm}$ in one dimension (see figure $\mathbf{2 A}$ ).
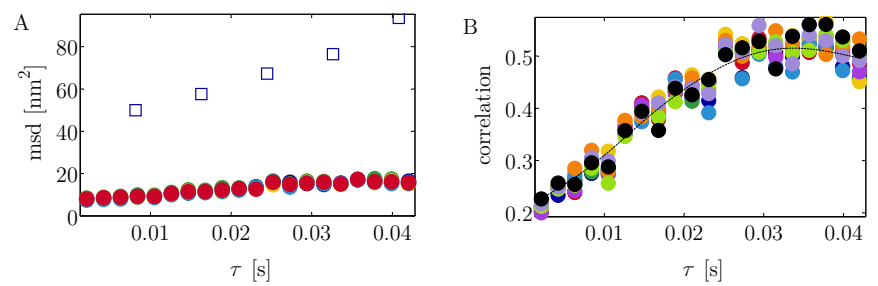

Fig. 2. A) Mean square displacements of 5 beads glued on a glass substrate $(\bullet)$ and typical mean square displacement measured in a cell for the same time lags ( $\square)$. B) Correlation between the displacements of each pair of beads glued on the substrate.

To complete this procedure it is generally necessary to remove a static error given by the mean square displacements (MSD) of beads glued on a glass substrate (figure 2A), The high correlation between the displacement of these beads (figure 2B) reveals that the static error is largely due to the vibration of the sample in the frame of the camera, because of the limited, though high, rigidity of the microscope.

\subsubsection{Cell mechanics and cell activity}

The local rheological properties of the cell cytoskeleton can be extracted from the MSD $\left\langle\Delta r^{2}(\tau)\right\rangle$ of the spherical particles using the Gener (GSER) $(19 ; 20)$ and payi $\sqrt{\bar{\sigma}}$ particular attention to the scaling of the energy which drives the beads motion in order to cope with the failure of the traditional fluctuation dissipation theorem (15). In order to avoid a Fourier transformation of the finite data series of the MSD we use here a time domain representation of the GSER to relate the $\left\langle\Delta r^{2}(\tau)\right\rangle$ to the local creep compliance $J(\tau)$ of the cytoskeleton, where $\tau$ is the lag time:

$$
\left\langle\Delta r^{2}(\tau)\right\rangle=\frac{2 k_{b} T_{\varepsilon}}{3 \pi a} J(\tau)
$$

where $a$ is the radius of the particles and $k_{b}$ Boltzmann's constant. Unlike the absolute temperature $T$ of the standard GSER, the temperature $T_{\varepsilon}$ considered here is an effective temperature. The notion of effective temperature has been recently introduced by Wilhelm (14) so that the energy $k_{b} T_{\varepsilon}$ incorporates the non-thermal contribution generated by any activity of the cell adhered to the substrate (21). At small time lags, well below the smallest characteristic time scale of the cell activity, there is no deviation from equilibrium and the particles in the cell are under pure Brownian motion scaled by energy $k_{b} T$. With increasing time lag, the cell activity starts to provide a 
supplementary source of energy which contributes to the particles' motion. At larger time lags, the thermal contribution become negligible compared with the cell contribution. In this regime, for time lags lying between $10^{-1}$ and $10 \mathrm{~s}$, Wilhelm shows the energy follows a power law $k_{b} T_{\varepsilon} \propto \tau^{\gamma}$, with exponent $\gamma$. This result is consistent with measurements carried out by several authors on different types of adherent cells $(15 ; 21)$. Considering the two separate sources of energy which may contribute to the bead motion, we propose here to write the effective temperature in the form:

$$
T_{\varepsilon}(\tau)=T\left(1+\left(\frac{\tau}{\tau_{c}}\right)^{\gamma}\right)
$$

with $\gamma>0$ and $\tau_{c}$ a cut-off time scale, such that for time lags $\tau \ll \tau_{c}$, the particle motion depends only on the thermal energy $k_{b} T$, whereas for time lags $\tau \gg \tau_{c}$ the particles motion depends mainly on the cell activity. The relation (2) is only valid if the thermal forces and the intracellular active forces are uncorrelated, which would generally be expected to be the case.

To complete this picture we utilise the growing evidence that the ability of the cell cytoskeleton to deform follows a power law over a broad range of time scales, at least between $10^{-3}$ to $10 \mathrm{~s}(22 ; 23 ; 24)$. If considered as a soft glassy material, the cell cytoskeletal rheology can be described by the soft glassy rheology (SGR) model (6). The SGR model is based on an effective noise temperature $x$, used to distinguish glassy behaviour for $x<1$, from a viscoelastic behaviour for $1<x<2$ and a pure Newtonian fluid beyond $x=2$. Again, in the framework of this model the creep compliance follows a power law when the cell cytoskeleton is mechanically probed in the linear regime (25): $J(\tau) \propto \tau^{\alpha}$, with the exponent $\alpha=x-1$ in the regime $1<x<2$. One can move from a purely elastic behaviour if $x=1, J(\tau) \propto t^{0}$, to increasingly viscous behaviour when $x$ increases, and reach purely fluid behaviour if $x=2, J(\tau) \propto t^{1}$. The creep compliance is here written:

$$
J(\tau)=J_{o}\left(\frac{\tau}{\tau_{c}}\right)^{\alpha},
$$

where $\alpha$ lies between 0 and 1 . The creep compliance $J_{o}$ is obtained when the time lag equals the cutoff time scale $\tau_{c}$.

Consequently, the MSD takes the form:

$$
\left\langle\Delta r^{2}(\tau)\right\rangle=J_{o} \frac{2 k_{b} T}{3 \pi a}\left(\left(\frac{\tau}{\tau_{c}}\right)^{\alpha}+\left(\frac{\tau}{\tau_{c}}\right)^{\beta}\right),
$$

with $\beta=\alpha+\gamma$. As $\alpha<\beta$, the motion is indeed purely Brownian for $\tau \ll \tau_{c}$ and purely controlled by the cell activity for $\tau \gg \tau_{c}$. This relation is particularly useful as it allows us to distinguish the contribution of the pure thermal noise scaled with exponent $\alpha$, from the contribution scaled with exponent $\beta$ due to the cell's own activity.

\section{Results and Discussion}

\subsection{Average and local properties}

\subsubsection{Rheological impact of the cell substrate interactions}

We will start by comparing the response of adhered and non-adhered adherent cells. We can see immediately the importance of the substrate on the cell behaviour, in figure 3, which depicts measurements carried out within a cell adhered to a glass substrate (lower part of the figure) and within a cell essentially in suspension but held at one point to a fibre (upper part of the figure). There are clear differences, qualitatively as well as quantitatively, in the shapes of the curves from beads in the two types of cell. Measurement carried out on different beads distributed within the adhered cell reveal a range of curves. Each of these is extremely well fitted by the relation (4). It is worth mentioning here that each of the parameters of the relation (4) can be easily identified as it controls a specific feature of the MSD curve in a log log plot. The exponents $\alpha$ and $\beta$ are respectively imposed by the slope at small time lag and large time lag, while $J_{o}$ controls only the position of the curve on the vertical axis and $\tau_{c}$ the position of the transition shoulder between the short and long time behaviour. For each of these parameters, the mean values and the standard deviations were calculated from the set of individual values identified from each experimental curve:

$$
\begin{aligned}
J_{o} & =5.210^{-2} \pm 1.510^{-2} \mathrm{~Pa}^{-1}, \\
\alpha & =0.41 \pm 0.09 \\
\beta & =1.21 \pm 0.24 \\
\tau_{c} & =46 \pm 10 \mathrm{~ms} .
\end{aligned}
$$

The mean values are in very good agreement with values found in the literature $(14 ; 15)$. Especially the value obtained for $\alpha$ confirms the cell contents are fully viscoelastic. Though each curve can be well fitted by the relation (4), there is a spread in the actual values which fit the data. This spread is greatest for $J_{o}$ and the variation indicates the heterogeneity in the cell environment. The excess kurtosis (not shown) for each of these parameters is around -1 indicating values rather uniformly spread around the mean. Further work would be needed to establish how this local variation relates to the position of the bead within the cell, including the distance from the nucleus.

In contrast, the cell in suspension behaves completely differently. This non-adhering cell is tightly fixed through a single point to an adhered cell spread along a rigid fiber ${ }^{1}$ slightly tilted above the substrate and one end of which is adhered to the substrate. Unlike the cells adhered to the rod itself, which exhibit an elongated shape, the suspended cell is almost completely spherical. The MSD of particles trapped in this particular cell is striking. One

1 This fibre detached from a cleaning paper wept against the substrate before the beginning of the experiment 


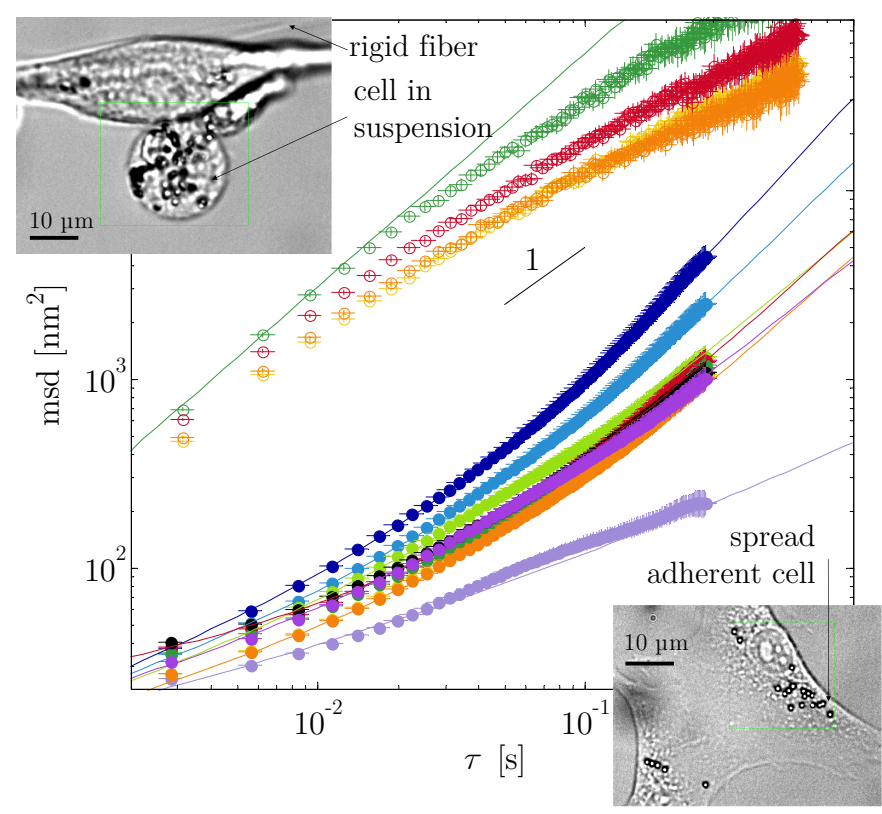

Fig. 3. MSD of beads trapped in a cell in suspension (open symbols, upper part of the graph), and MSD of beads trapped in an adhered cell (closed symbols lower part of the graph. Solid lines are fits of equation 4.) The images show respectively the non-adhered cell (upper image) and the spread, adhered cell (lower image).

observes a concave MSD profile with MSD values approximately ten times greater than the typical convex profile obtained for the adhered cell on this range of time scales. The suspended cell is consequently much softer than the adhered cells. Moreover, the slope of the MSD curve for the smallest time lags is around 1.2. This superdiffusive behaviour suggest first that the cell is maybe more fluid, and second that it is a highly active environment at time scales much shorter that those observed in the adhered cell $(21 ; 26)$. As a matter of fact the model (4) fails to fit this particular behaviour, as can be seen by the deviation from the solid line. The lack of fit with our model for the non-adhered cell is not surprising as the two important hypothesis $\equiv$ ur madgl are not verified: it is neither proven that the $\overline{\overline{\bar{F}}}$ pended cell behave $\lambda$ as a soft glassy material nor that the cell activity scaling is valid on the same frequency range as the one observed for adhered cell. Indeed it seems that the cell activity as an impact at time lags much smaller than for an-adhered cell which could mean that intracellular phenomena are faster than those observed in an adhered cell. As a result $\mathrm{f} \equiv$ tures observed in adhered cell at given time lags should be observed at shorter time lags in the nonadhered cell. Typically the decrease of the slope of the MSD for adhered cell at time lags larger than $10 \mathrm{~s}$ (for instance figure $5 \mathrm{C}$ of (15)), decrease which is out of the range of our model, could be observed much earlier in the active non-adhered cell.
This could explain the shape of the our curves for the non-adhered cell.

\subsubsection{A heterogeneous isotropic material}

$\equiv$ re 4 shows for each bead incorporated in a spread adFrered cell the magnitude of the MSD, according to the direction of the displacement, for two typical time lags. In both cases, the trajectories are circular. This indicates no particular angular dependence and consequently no noticeable anisotropy in the local response of the cell. In the absence of directional stimuli, the cells behave as an isotropic material $(27 ; 28)$.

However, the cell cytoskeleton is a non-uniform material. As further illustrated in figure 5, the MSD of beads incorporated within the cell depends strongly on the position of the beads at short time lags as well as at long time lags. Beyond these regional differences at the scale of the cell, it is worse $\wedge$ remembe $\equiv$ that strong variations may occur from one cell to aner, according to the age of the cell, its phase in the life cycle, the number of passages, but also according to subtle changes in the cell environment such as temperature and $\mathrm{pH}$ changes.

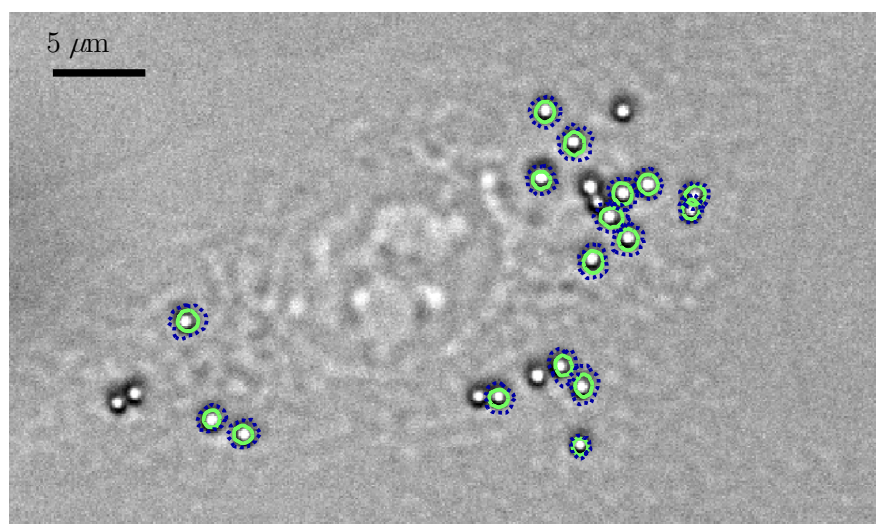

Fig. 4. Isotropy of the cell. Angular distribution of the $\sqrt{\mathrm{MSD}}$ for two different time lags: $(-) 10^{-2} \mathrm{~s},(\bullet) 5.10^{-2} \mathrm{~s}$. The actual $\sqrt{\mathrm{MSD}}$ have been magnified 30 times in the figure for clarity).

\subsection{Moving away from the physiological environment}

Numerous works have focused on the mechanical response of cells exposed to mechanical perturbations such as laminar shear stresses $(27)$, stretching $(2)$ or traction $(23 ; 29)$. However the mechanical response of cells due to variations in temperature and $\mathrm{pH}$ away from physiological values has been rarely investigated and never in real time to reveal the transient response $(16 ; 17)$. Throughout our series of experiments, changing the temperature or the $\mathrm{pH}$ on a limited time scale of around one hour never induced noticeable changes in the cells' external shape and hence 


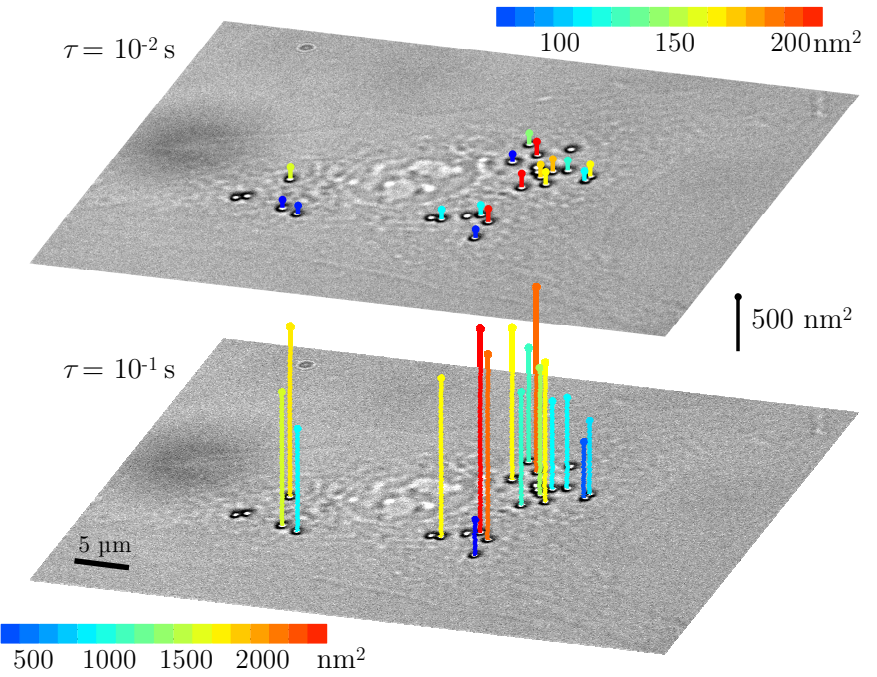

Fig. 5. Heterogeneity in the cell. The local MSD are indicated by the height of the vertical lines for two different time lags: $10^{-2} \mathrm{~s}$ upper figure, $10^{-1} \mathrm{~s}$ lower figure.

there was no macroscopic signature which could have revealed the strong perturbations of the cell rheology and cell activity we have measured.

\subsubsection{Rheological response to temperature changes}

Adherent cells grown and spread on a glass substrate in the microincubatowere submitted to cycles of temperature changes. $\mathrm{A} \overline{\overline{\bar{\sigma}}}$ e comprises a $4 \mathrm{~min}$ temperature drop of $10^{\circ}$ from the physiological value of $37^{\circ} \mathrm{C}$, a period of stabilisation of $20 \mathrm{~min}$ at a temperature of $27^{\circ} \mathrm{C}$ and a 4 min temperature increase back to $37^{\circ} \mathrm{C}$. During this process the displacement of the beads incorporated in the cell were continuously tracked. Because of the temperature variations, the microincubator is alternatively subjected to a slight dilation ${ }_{\lambda}$ which modifies the vertical position of the cells. To compensate this phenomenon, the focal plane is regularly readjusted manually while the temperature is changing, to keep a clear image of the cell. Nevertheless, for each vertical position the beads shape may slightly change. An independent filter is then selected for each bead and for each temperature of interest to ensure optimum tracking.

In order to exhibit the response most clearly, in figure 6 features $\bar{\equiv}$ oncentrate on a single bead-situated in the vicinity of the nucleus. All beads show a similar response, but averaging the time dependent MSD of all the beads simply blurs the interesting local features that can be deduced from the study of the MSD of a single bead. As shown in figure 6, one notices a clear decrease of the MSD when the temperature drops. The general response is the same for all the beads, although the amplitude and the duration of the transient regime vary from one bead to another.

While the process seems fully reversible after one cycle of temperature decrease and increase, corresponding

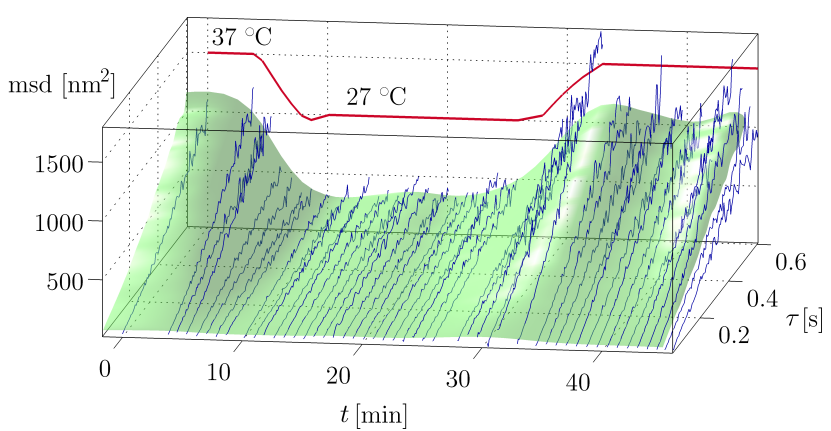

Fig. 6. Typical time evolution of the MSD according to the temperature for one bead at a given locus in an spread cell: - experimental MSD, - temperature. The surface is a visual guide.
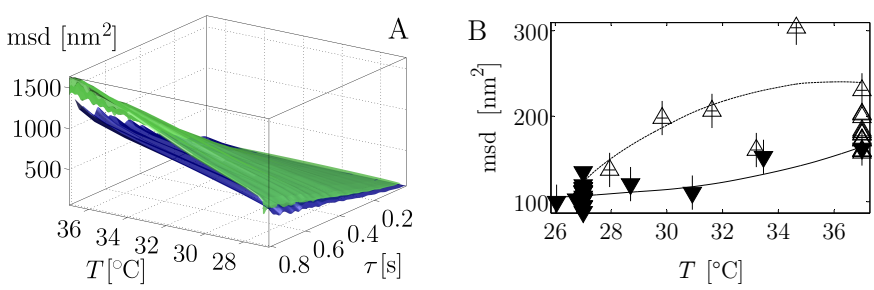

Fig. 7. A): temperature dependence of the experimental MSD during the cooling process (dark blue surface) and the heating process (light green surface). B): cut of the surface for a time lag of $0.1 \mathrm{~s}, \boldsymbol{\nabla}$ MSD during the cooling, $\triangle$ MSD during the heating, solid lines are visual guides.

to a one hour measurement series, it clearly appears on the figure 7 that the 'rejuvenation' process observed when the cell returns to its physiological condition, and hence presumed 'normal' behaviour, differs from the process observed when the cell is adapting to a non-physiological environment. One especially notices that the rejuvenation process ends up with an overshoot which is progressively damped to finally achieve a return to the initial response of the cell.

In order to further understand this hysteretic process each of the MSD curves obtained at various temperatures has been fitted with the model (4). Again the model fits perfectly all the curves measured in the stabilised-state as well as in the transient regime. Figure 8 shows the averaged stabilised-state MSD for the particular bead of interest at $37^{\circ} \mathrm{C}$ and $27^{\circ} \mathrm{C}$ and the MSD observed at two different typical temperatures during the cooling process (temperatures followed by a - exponent) and during the heating process (temperatures followed by a + exponent).

Despite the changing shapes of the curves measured throughout the temperature cycle, we found that all of them can be well fitted using the same $\alpha=0.09 \pm 0.01$ and $\beta=1.2 \pm 0.02$ exponents. This indicates that the local viscoelasticity and the local cell activity scalings are preserved throughout the temperature cycle. The evolution 


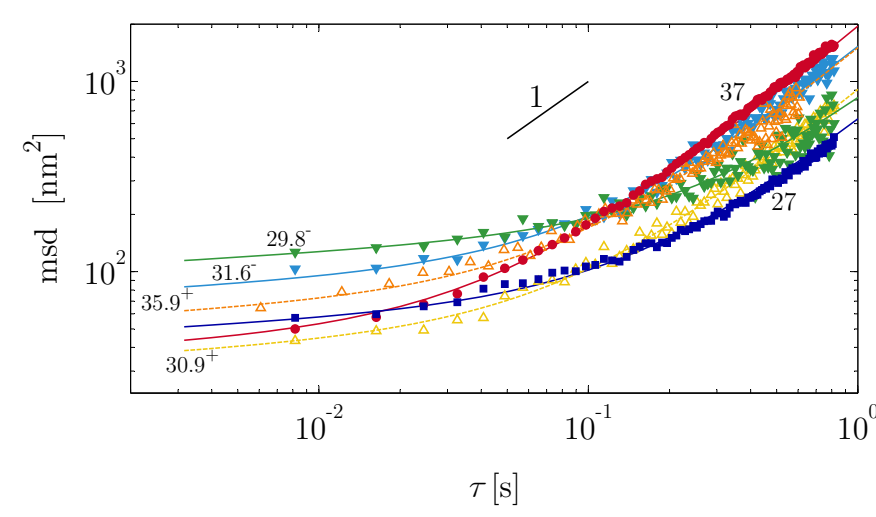

Fig. 8. Evolution of the transient MSD profiles during the cooling $(\boldsymbol{\nabla})$ and heating $(\triangle)$ processes. - stabilised-state behaviour at $37^{\circ} \mathrm{C}$, stabilised-state behaviour at $27^{\circ} \mathrm{C}$. Temperature is indicated beside each curve, followed by a - exponent and $\mathrm{a}+$ exponent respectively for the cooling and the heating processes.

of the MSD depends only on a change in the cutoff time scale $\tau_{c}$ and the creep compliance $J_{o}$ (see figure 9 ).
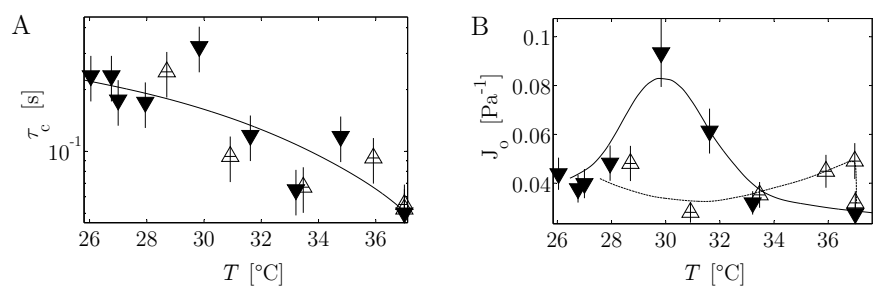

Fig. 9. A: temperature dependence of $\tau_{c}$. B: temperature dependence of $J_{o} . \boldsymbol{\nabla}$ cooling process, $\triangle$ heating process, solid lines are visual guides.

We found indeed that $\tau_{c}$ follows a monotonic increase during the cooling process, its value at $27^{\circ} \mathrm{C}$ beeing four times larger than the usual value of $50 \mathrm{~ms}$ found at $37^{\circ} \mathrm{C}$. Unlike $\tau_{c}$ which seems to follow the same path during the cooling and heating process, the parameter $J_{o}$ exhibits a non-reversible behaviour, with non-monotonic evolution during the cooling and heating process. This particular behaviour is linked to the hysteresis exhibited by the MSD. Interestingly $J_{o}$ is approximately $50 \%$ larger at $27^{\circ} \mathrm{C}$ than at $37^{\circ} \mathrm{C}$. To be able to compare the creep compliances $J$ observed at these two temperatures for the same time lags, it is necessary to evaluate the ratio $J_{o} / \tau_{c}^{\alpha}$ introduced in the relation 3 . Due to the small value of $\alpha, J_{o} / \tau_{c}^{\alpha}$ is larger at $27^{\circ} \mathrm{C}$ than at $37^{\circ} \mathrm{C}$ even if $\tau_{c}$ drops with the temperature. This means, that the cytoskeleton is effectively softer at $27^{\circ} \mathrm{C}$ than at $37^{\circ} \mathrm{C}$ even if the MSD is globally smaller at $27^{\circ} \mathrm{C}$ than at $37^{\circ}$, due to the slow down of cell activity indicated by the shift of $\tau_{c}$ to larger value when the temperature is decreased.

\subsubsection{Rheological response to $\mathrm{pH}$ changes}

The $\mathrm{pH}$ environment of the cell was suddenly changed by flushing a solution maintained at $37^{\circ} \mathrm{C}$ in contact with a $0 \% \mathrm{CO}_{2}$ atmosphere into the microincubator chamber. The normal situation is restored by flushing a second solution maintained at $37^{\circ} \mathrm{C}$ in contact with a $5 \% \mathrm{CO}_{2}$ atmosphere. As illustrated on the figure 10, A drastic change in the MSD is observed between the normal and the anomalous (non-physiological) situation. More striking, the cell goes back to its initial state when replaced into its physiological environnement This aging-rejuvenation process is thus reversible.

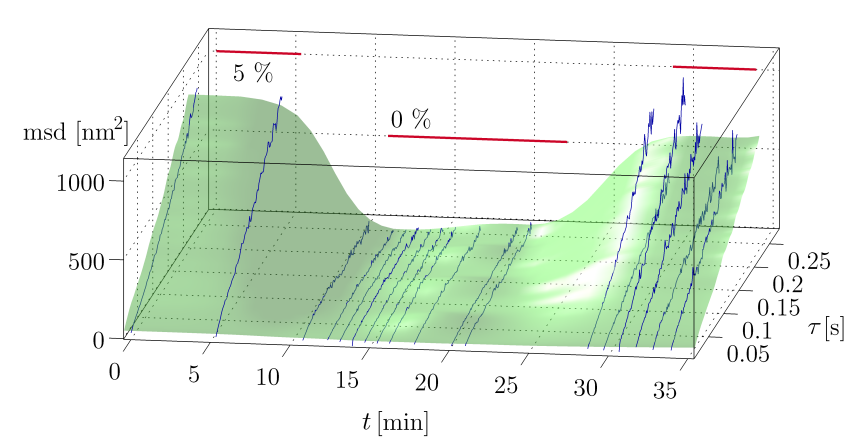

Fig. 10. Time evolution of the MSD according to the $\mathrm{CO}_{2}$ percentage: - experimental MSD, $-\mathrm{CO}_{2}$ percentage. The surface is a visual guide.

Figure 11 shows the average MSD observed locally for $5 \% \mathrm{CO}_{2}$ and for $0 \% \mathrm{CO}_{2}$. The values identified from the model fit, reveal now that $\tau_{c}=50 \mathrm{~ms}$ in both cases suggesting that $\tau_{c}$ could be mainly controlled by the temperature. On the other hand the other parameters are strongly affected by the change of $\mathrm{pH}$ :

$$
\begin{gathered}
J_{o}(5 \%)=5.210^{-2} \mathrm{~Pa}^{-1} \quad \alpha(5 \%)=0.25 \quad \beta(5 \%)=1.35 \\
J_{o}(0 \%)=1.710^{-2} \mathrm{~Pa}^{-1} \quad \alpha(0 \%)=0.12 \quad \beta(\%)=0.55 .
\end{gathered}
$$

We observed a moderate increase of $J_{o}$ when the temperature decreases from the physiological level, The strong decrease of $J_{o}$ when the $\mathrm{pH}$ increases from the physiological level indicates this time a clear stiffening of the cytoskeleton with the alkalinization of the environment and an increased osmotic stress. The drop in the parameter $\beta$ also reveals a reduction in the amplitude of the cellular activity with no noticeable slow down in the kinetics of the active processes.

\section{Conclusions}

One-point microrheometric measurements carried out on cells cultured in a microincubator have been carried out to study the impact of a temperature change and a $\mathrm{pH}$ 


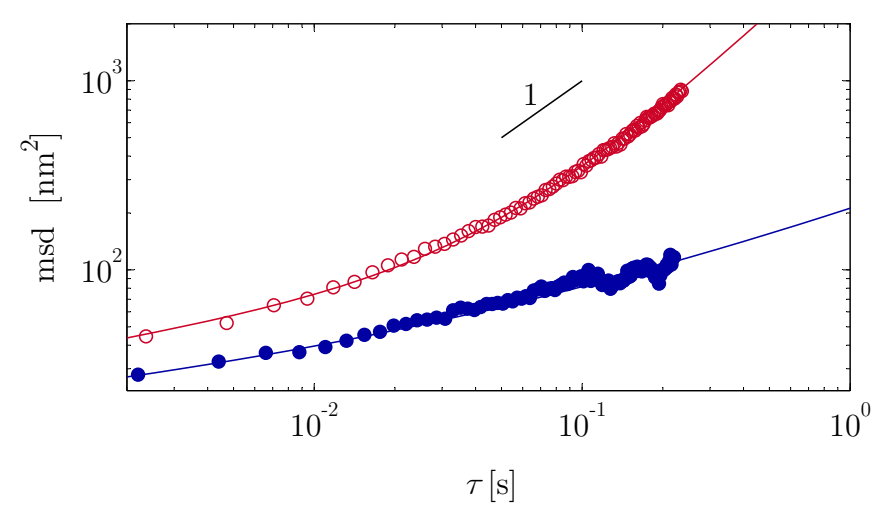

Fig. 11. MSD profiles at normal $\mathrm{pH}\left(5 \% \mathrm{CO}_{2}, \bigcirc\right)$ and high $\mathrm{pH}\left(0 \% \mathrm{CO}_{2}, \bullet\right)$. Solid lines are fits of the relation (4).

change on the local rheology of the cytoskeleton of cells allowed to adhere and spread on a glass substrate. Firstly, in a physiological environment, it is clear there are very substantial differences in response for adhered and nonadhered adherent cells. The cytoskeleton of an adhered cell is indeed much stiffer than the cytoskeleton of a nonadhered cell. When the environment is changed, our results reveal a fast, strong but reversible dependence on temperature and $\mathrm{pH}$. For uncorrelated thermal forces and intracellular active forces we introduce a modified generalised Stokes-Einstein equation to take into account the impact of the cell activity on the tracer motion used for the microrheometry. Measurements analysed with this relation suggest that the cell becomes softer and that its activity is slowed down when the temperature decreases. The respective exponents of the power laws characterising the cell rheology and the cell activity are however preserved throughout the temperature variation. The hysteresis observed during the aging/rejuvenation process could be explained by the non-monotonic behaviour of the creep compliance prefactor. A sudden change in $\mathrm{pH}$ gives also rise to anomalous rheologic behaviour, with a stiffening of the cytoskeleton when the culture medium becomes more alkaline. These results confirms that in vitro experiments have to be carried out in a well controlled environment in order get meaningful results. The absence of visual changes in the cell's shape can clearly hide dramatic changes in the cell behaviour, at least over time periods of several tens of minutes.

We would like to thank Sheila McNeil, Giuseppe Batt Bals $\overline{\overline{\bar{\sigma}}}$ or the milling of the micro-incubator and his help concerning its design. EPSRC for funding of this project.

\section{References}

1. M.R. Mofrad, Annual Review of Fluid Mechanics 41(1) (2009)
2. X. Trepat, L.H. Deng, S.S. An, D. Navajas, D.J. Tschumperlin, W.T. Gerthoffer, J.P. Butler, J.J. Fredberg, Nature 447(7144), 592 (2007)

3. A.R. Bausch, K. Kroy, Nat Phys 2(4), 231 (2006), ISSN 1745-2473

4. D.E. Discher, P. Janmey, Y.L. Wang, Science 310(5751), 1139 (2005)

5. L.H. Deng, X. Trepat, J.P. Butler, E. Millet, K.G. Morgan, D.A. Weitz, J.J. Fredberg, Nature Materials $\mathbf{5}(8), 636(2006)$

6. K.K. Mandadapu, S. Govindjee, M.R. Mofrad, Journal of Biomechanics 41(7), 1467 (2008), ISSN 00219290

7. P. Bursac, G. Lenormand, B. Fabry, M. Oliver, D.A. Weitz, V. Viasnoff, J.P. Butler, J.J. Fredberg, Nature Materials 4(7), 557 (2005)

8. J. Solon, I. Levental, K. Sengupta, P.C. Georges, P.A. Janmey, Biophys. J. 93(12), 4453 (2007)

9. K.E. Kasza, A.C. Rowat, J. Liu, T.E. Angelini, C.P. Brangwynne, G.H. Koenderink, D.A. Weitz, Current Opinion in Cell Biology 19(1), 101 (2007), ISSN 09550674

10. C. Moraes, C.A. Simmons, Y. Sun, CSME BULLETIN SCGM pp. 15-18 (2006)

11. S.R. Heidemann, D. Wirtz, Trends in cell biology 14, 160 (2004)

12. B.R. Daniels, B.C. Masi, D. Wirtz, Biophysical Journal 90(12), 4712 (2006)

13. E.M. Vinck, B.J. Cagnie, M.J. Cornelissen, H.A. Declercq, D.C. Cambier, Photomedicine and Laser Surgery 23, 167 (2005)

14. C. Wilhelm, Physical Review Letters 101(2), 028101 (2008)

15. B.D. Hoffman, G. Massiera, K.M. Van Citters, J.C. Crocker, PNAS 103, 10259 (2006)

16. M. Jonas, H.D. Huang, R.D. Kamm, P.T.C. So, Biophysical Journal 95(2), 895 (2008)

17. K.S. Wu, W.W. van Osdol, R.H. Dauskardt, Biomaterials 27(5), 785 (2006)

18. M.T. Valentine, P.D. Kaplan, D. Thota, J.C. Crocker, T. Gisler, R.K. Prud'homme, M. Beck, D.A. Weitz, Phys. Rev. E 64, 061506 (2001)

19. T.G. Mason, Rheol. Acta 39, 371 (2000)

20. T. Mason, D.A. Weitz, Phys. Rev. Lett. 74, 1250 (1995)

21. A.W.C. Lau, B.D. Hoffman, A. Davies, J.C. Crocker, T.C. Lubensky, Phys. Rev. Lett. 91(19), 198101 (2003)

22. G. Lenormand, P. Bursac, J.P. Butler, J.J. Fredberg, Physical Review E 76(4), 041901 (2007)

23. M. Balland, N. Desprat, D. Icard, S. Fereol, A. Asnacios, J. Browaeys, S. Henon, F. Gallet, Physical Review E 74(2), 021911 (2006)

24. G. Lenormand, E. Millet, B. Fabry, J.P. Butler, J.J. Fredberg, Journal Of The Royal Society Interface 1(1), 91 (2004)

25. P. Sollich, in Molecular Gels: Materials with SelfAssembled Fibrillar Networks (Springer, Dordrecht, 2006), pp. 161-192 
26. A. Caspi, R. Granek, M. Elbaum, Phys. Rev. Lett. 85(26), 5655 (2000)

27. J.C. del Alamo, G.N. Norwich, Y.S.J. Li, J.C. Lasheras, S. Chien, Proceedings Of The National Academy Of Sciences Of The United States Of America 105(40), 15411 (2008)

28. I.A. Hasnain, A.M. Donald, Phys. Rev. E 73(3), 031901 (2006)

29. N. Desprat, A. Richert, J. Simeon, A. Asnacios, Biophys. J. 88(3), 2224 (2005) 